\title{
OPTIMIZATION OF SOLVENT FLOW PATTERN FOR EXTRACTIVE REACTORS IN SERIES*
}

\author{
Shigeo GOTO AND MASAKazu MATSUBARA \\ Department of Chemical Engineering, Nagoya University, Nagoya
}

\begin{abstract}
The optimal solvent flow pattern for multistage reactors with extraction is investigated. The general structure is considered by introducing structure variables and is optimized by use of the method of Goldfarb et al. Numerical calculations are carried out for parameter values tentatively chosen. The results indicate some basic features of the optimal solvent flow pattern. Some qualitative considerations suggest that these features will probably be the general properties of the optimal pattern.
\end{abstract}

\section{Introduction}

In conventional chemical process design, separate equipment used to be provided for each chemical reaction and each physical unit operation. But if the desired products are intermediates of complex reactions which may be rapidly converted into wasteful final products, the performance of the process can be improved by the selective removal of the desired product from the reaction system. In other words, simultaneous operation which is a combination of chemical reaction and physical unit operation in one piece of equipment may be more profitable than successive operations in separate equipment.

One such operation is the reaction with extraction, which was referred to as extractive reaction by Piret et al. ${ }^{7)}$ and was applied to the process of furfural formation by Hofmann ${ }^{5)}$ and Schoenemann ${ }^{8)}$. Recently the authors studied the effects of extraction rate and reactor type on the performance of the extractive $^{\text {reactor }}{ }^{2)}$ and on the optimization of an extractive stirred-tank reactor coupled with separators in three different fashions ${ }^{3}$.

In this paper, the optimal structure problems are studied with respect to the solvent flow pattern for multistage continuous stirred-tank reactors in which extractive reactions are carried out.

The techniques for optimizing the system structure which have heretofore been proposed can be classified into three categories ${ }^{4,6}:$ 1) previously establishing fundamental rules about the optimal structure, 2) the heuristic evolution and 3) the optimization of general structure containing structure variables. The first technique is mainly applicable to the homogeneous system which is composed of subsystems

\footnotetext{
* Received on September 4, 1973

干464 名古屋市千種区不老町

名古屋大学工学部化学工学教室 松原正一
}

having similar functions, while the second is applicable to the heterogeneous system which is composed of subsystems having different functions. The third is useful for both homogeneous and heterogeneous systems.

The system considered in this paper is a homogeneous system which is composed of heterogeneous subsystems having two different functions, that is, the reaction and the extraction. The general structure including all the possible couplings between subsystems is considered by introducing structure variables. The system having the general structure is then optimized by use of the method due to Goldfarb et al. ${ }^{11}$ to seek the optimal structure. This method is an extension of the method of Davidon, Fletcher and Powell for nonlinear programming problems involving linear constraints of equalities and inequalities.

\section{Problem Formulation}

$N$ continuous stirred-tanks in series are used for the extractive reaction. The solvent $\mathrm{S}_{1}$ containing the raw material flows in cascade from the first to the $N$-th tank, while the solvent $\mathrm{S}_{2}$ for the extraction flows between $N$ tanks in all the possible fashions.

The following conditions are assumed to formulate the state equations of the $n$-th subsystem, which is the $n$-th extractive reactor shown in Fig. 1.

(i) Two solvents, $S_{1}$ and $S_{2}$, are insoluble in each other and therefore form two phases. The reactions

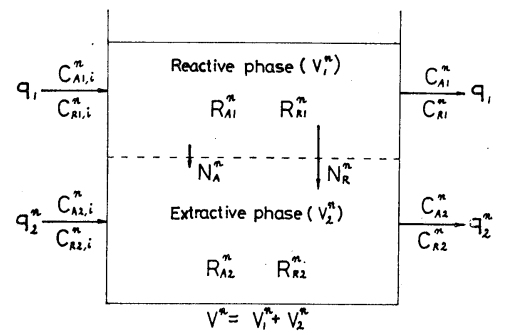

Fig. 1 A hypothetical extractive stirred tank reactor 
primarily proceed in $S_{1}$ and the desired product is extracted by $S_{2}$. The phases of $S_{1}$ and $S_{2}$ are referred to as the reactive phase and the extractive phase, respectively.

(ii) Each stirred-tank reactor is followed by a separator. Two phases are well mixed in each other in the stirred-tank reactor. One is a dispersed phase and the other is a continuous phase. They are then perfectly separated in the subsequent separator.

(iii) The reaction is a combination of reversible and consecutive reactions which are of the first order, that is,

$$
\mathrm{A} \underset{k^{\prime}}{\stackrel{k}{\rightleftarrows}} \mathrm{R} \stackrel{k^{\prime \prime}}{\longrightarrow} \mathrm{S}
$$

where $\mathrm{R}$ is the desired product and $\mathrm{S}$ the wasteful product.

The reaction rates are

$$
\begin{aligned}
& R_{\Lambda 1}^{n}=-k_{1} C_{\Lambda 1}^{n}+k_{1}^{\prime} C_{\mathrm{R} 1}^{n} \\
& R_{\mathrm{R} 1}^{n}=k_{1} C_{\Lambda 1}^{n}-\left(k_{1}^{\prime}+k_{1}^{\prime \prime}\right) C_{\mathrm{R} 1}^{n}
\end{aligned}
$$

for the reactive phase and

$$
\begin{aligned}
& R_{A 2}^{n}=-k_{1} C_{A 2}^{n}+k_{2}^{\prime} C_{R 2}^{n} \\
& R_{R 2}^{n}=k_{2} C_{A 2}^{n}-\left(k_{2}^{\prime}+k_{2}^{\prime \prime}\right) C_{R 2}^{n}
\end{aligned}
$$

for the extractive phase, where the reaction rate constants for the reactive phase are large relative to those for the extractive phase.

(iv) The concentrations of $\mathrm{A}$ and $\mathrm{R}$ are dilute in both phases, and consequently the distribution coefficients are constant regardless of concentration. Therefore,

$$
\left(C_{\mathrm{A} 2}^{n}\right)^{*}=m_{\mathrm{A}} C_{\mathrm{A} 1}^{n}, \quad\left(C_{\mathrm{R} 2}^{n}\right)^{*}=m_{\mathrm{R}} C_{\mathrm{R} 1}^{n}
$$

where $m_{\mathrm{R}}$ is large relative to $m_{\mathrm{A}}$.

(v) The mass transfer rates of $\mathrm{A}$ and $\mathrm{R}$ from the reactive to the extractive phase are

$$
\begin{aligned}
& N_{\mathrm{A}}^{n}=k_{\mathrm{A}}^{n} a\left(\left(C_{\mathrm{A} 2}^{n}\right)^{*}-C_{\mathrm{A} 2}^{n}\right)=k_{\mathrm{A}}^{n} a\left(m_{\mathrm{A}} C_{\mathrm{A} 1}^{n}-C_{\mathrm{A} 2}^{n}\right) \\
& N_{\mathrm{R}}^{n}=k_{\mathrm{R}}^{n} a\left(\left(C_{\mathrm{R} 2}^{n}\right)^{*}-C_{\mathrm{R} 2}^{n}\right)=k_{\mathrm{R}}^{n} a\left(m_{\mathrm{R}} C_{\mathrm{R} 1}^{n}-C_{\mathrm{R} 2}^{n}\right)
\end{aligned}
$$

(vi) Both phases are isothermal.

For the $n$-th continuous stirred-tank reactor, the equations of mass balance under assumptions (i)-(vi) are

$$
\begin{array}{r}
q_{1}\left(C_{A 1, i}^{n}-C_{A 1}^{n}\right)-N_{A}^{n} V^{n}+R_{A 1}^{n} V_{1}^{n}=0 \\
q_{1}\left(C_{\mathrm{R} 1, i}^{n}-C_{\mathrm{R} 1}^{n}\right)-N_{\mathrm{R}}^{n} V^{n}+R_{\mathrm{R} 1}^{n} V_{1}^{n}=0 \\
q_{2}^{n}\left(C_{A 2, i}^{n}-C_{A 2}^{n}\right)+N_{A}^{n} V^{n}+R_{\Lambda 2}^{n} V_{2}^{n}=0 \\
q_{2}^{n}\left(C_{\mathrm{R} 2, i}^{n}-C_{\mathrm{R} 2}^{n}\right)+N_{\mathrm{R}}^{n} V^{n}+R_{\mathrm{R} 2}^{n} V_{2}^{n}=0
\end{array}
$$

It can easily be shown under the assumption (ii) that $V_{1}^{n} / V_{2}^{n}$ becomes equal to $q_{1} / q_{2}^{n}$. Therefore, substitution of Eqs. (1)-(6) into Eqs. (7)-(10) and introduction of dimensionless groups indicated in the Nomenclature yield a set of state equations for the $n$-th subsystem.

\footnotetext{
* Though the structure variable is usually defined as $\alpha_{m n}=$ $\zeta_{m n} / \zeta^{m}, \zeta_{m n}$ is so called in this paper.
}

$$
\begin{aligned}
f_{1}^{n}= & \left(1+m_{\mathrm{A}} \xi_{\mathrm{A}}^{n} \tau^{n}+\frac{\tau^{n}}{1+\zeta^{n}}\right) x_{1}^{n}-\frac{\kappa_{\mathrm{R} 1} \tau^{n}}{1+\zeta^{n}} y_{1}^{n} \\
& -\xi_{\mathrm{A}}^{n} \tau^{n} x_{2}^{n}-x_{1, i}^{n}=0 \\
f_{2}^{n}= & -\frac{\tau^{n}}{1+\zeta^{n}} x_{1}^{n}+\left(1+m_{\mathrm{R}} \xi_{\mathrm{R}}^{n} \tau^{n}+\frac{\left(\kappa_{\mathrm{R} 1}+\kappa_{\mathrm{C} 1}\right) \tau^{n}}{1+\zeta^{n}}\right) y_{1}^{n} \\
& -\xi_{\mathrm{R}}^{n} \tau^{n} y_{2}^{n}-y_{1, i}^{n}=0 \\
f_{3}^{n}= & -m_{\mathrm{A}} \xi_{\mathrm{A}}^{n} \tau^{n} x_{1}^{n}+\left(\zeta^{n}+\xi_{\mathrm{A}}^{n} \tau^{n}+\kappa \frac{\zeta^{n}}{\left.1+\zeta^{n} \tau^{n}\right) x_{2}^{n}}\right. \\
& -\frac{\kappa \kappa_{\mathrm{R} 2} \zeta^{n} \tau^{n}}{1+\zeta^{n}} y_{2}^{n}-\zeta^{n} x_{2, i}^{n}=0 \\
f_{4}^{n}= & -m_{\mathrm{R}} \xi_{\mathrm{R}}^{n} \tau^{n} y_{1}^{n}-\frac{\kappa \zeta^{n} \tau^{n}}{1+\zeta^{n}} x_{2}^{n}+\left(\zeta^{n}+\xi_{\mathrm{R}}^{n} \tau^{n}\right. \\
& \left.+\frac{\kappa\left(\kappa_{\mathrm{R} 2}+\kappa_{\mathrm{C} 2}\right) \zeta^{n} \tau^{n}}{1+\zeta^{n}}\right) y_{2}^{n}-\zeta^{n} y_{2, i}^{n}=0
\end{aligned}
$$

Though a number of parameters are involved in Eqs. $(7)^{\prime}-(10)^{\prime}$, some of them, that is, $m_{\mathrm{A}}, m_{\mathrm{R}}, \kappa, \kappa_{\mathrm{R} 1}$, $\kappa_{\mathrm{R} 2}, \kappa_{\mathrm{C} 1}$ and $\kappa_{\mathrm{C} 2}$ will be fixed for the specified reaction system and the specified solvents, $\mathrm{S}_{1}$ and $\mathrm{S}_{2}$.

Extractive reaction is especially useful for a reaction system in which the desired intermediate may be rapidly converted into wasteful products unless it is immediately taken away. In other words, extractive reaction will be effective in situations where the values of $\kappa_{\mathrm{R} 1}, \kappa_{\mathrm{R} 2}, \kappa_{\mathrm{C} 1}$ and $\kappa_{\mathrm{C} 2}$ are relatively large.

To enhance the yield of the desired product by immediate extraction, the solvent $S_{2}$ should be such that the value of $m_{\mathrm{R}}$ is as large as possible and, in addition, the value of $m_{\mathrm{A}}$ as small as possible. These points are, though quite natural, ascertained by the authors for the reversible reaction ${ }^{2}$.

From these points of view, the values of parameters for the numerical calculations were chosen as $\kappa_{\mathrm{R} 1}=\kappa_{\mathrm{R} 2}=9, \kappa_{\mathrm{C} 1}=\kappa_{\mathrm{C} 2}=1, m_{\mathrm{R}}=10, m_{\mathrm{A}}=0.1$ and $\kappa=$ 0.01 . The concentrations of fresh feed are $x_{1}^{0}=1, y_{1}^{0}=0$ and $x_{2}^{0}=y_{2}^{0}=0$, that is, the feed solvent $\mathrm{S}_{1}$ contains only the reactant $\mathrm{A}$ while the feed solvent $\mathrm{S}_{2}$ is pure.

On the other hand, the values of $\tau^{n}, \xi_{A}^{n}, \xi_{R}^{n}$ and $\zeta^{n}$ are at our disposal when we want to optimize the whole system. But, since $\xi_{\mathrm{A}}^{n}$ and $\xi_{\mathrm{R}}^{n}$ depend on the stirring speed, they cannot be changed independently of each other. So assume that $\xi_{\mathrm{A}}^{n}=\xi_{\mathrm{R}}^{n} \equiv \xi^{n}$. Thus we have only three decision variables, $\tau^{n}, \xi^{n}$ and $\zeta^{n}$, for the $n$-th subsystem.

Consider all the possible flows of the solvent $\mathrm{S}_{2}$ between $N$ subsystems. Denote the normalized flow rates of these flows by structure variables $\zeta_{0 n}(n=1,2$, $\cdots, N)$ and $\zeta_{m n}(m=1,2, \cdots, N ; n=1,2, \cdots, N+1)$. The $\zeta_{0 n}$ is the flow rate of the fresh solvent $\mathrm{S}_{2}$ into the $n$-th subsystem, the $\zeta_{m n}(n \neq N+1)$ that of $\mathrm{S}_{2}$ from the $m$-th to the $n$-th subsystem and the $\zeta_{m, N+1}$ that of $\mathrm{S}_{2}$ from the $m$-th subsystem to the exhaust*. Figure 2 shows the structure of the whole system for $N=3$ in which all the possible flows of $S_{2}$ are incorporated. 
The feed concentrations of the $n$-th subsystem can be written as

$$
\begin{array}{ll}
x_{1, i}^{n}=x_{1}^{n-1} & n=1,2, \cdots, N \\
y_{1, i}^{n}=y_{1}^{n-1} & n=1,2, \cdots, N \\
x_{2, i}^{n}=\sum_{m=0}^{N} \zeta_{m n} x_{2}^{m} / \zeta^{n} & n=1,2, \cdots, N \\
y_{2, i}^{n}=\sum_{m=0}^{N} \zeta_{m n} y_{2}^{m} / \zeta^{n} & n=1,2, \cdots, N
\end{array}
$$

$\zeta^{n}$, which is the total normalized flow rate of $S_{2}$ into the $n$-th subsystem, must be equal not only to the sum of the entering flow rates but also to the sum of the leaving flow rates. Therefore the following equality constraints must be satisfied.

$$
\begin{array}{ll}
\zeta^{n}=\sum_{m=0}^{N} \zeta_{m n} & n=1,2, \cdots, N \\
\zeta^{n}=\sum_{m=1}^{N+1} \zeta_{n m} & n=1,2, \cdots, N
\end{array}
$$

Since flow rates must be non-negative, the following inequality constraints are imposed.

$$
\begin{array}{ll}
\zeta_{0 n} \geq 0 & n=1,2, \cdots, N \\
\zeta_{m n} \geq 0 & m=1,2, \cdots, N ; n=1,2, \cdots, N+1
\end{array}
$$

The realizable value of $\xi^{n}$ which is related to the stirring speed will be restricted. So, we tentatively assume the following inequality constraint for numerical calculations.

$$
1 \leq \xi^{n} \leq 100
$$

In the extractive reaction process design, the yield of the desired product from the extractive phase should be as large as possible while the total expenditure of the solvent $S_{2}$ should be as small as possible. So the value of the objective function

$$
\begin{aligned}
J & =\sum_{n=1}^{N} \zeta_{n, N+1} y_{2}^{n}-\lambda \sum_{n=1}^{N} \zeta_{0 n} \\
& =\sum_{n=1}^{N} \zeta_{n, N+1}\left(y_{2}^{n}-\lambda\right)
\end{aligned}
$$

should be maximized. The second expression can be derived from the relation $\sum_{n=1}^{N} \zeta_{0 n}=\sum_{n=1}^{N} \zeta_{n, N+1}$ which can easily be obtained by use of Eqs. (15) and (16).

The value of $\lambda$ in Eq. (19) which depends on the unit prices of the product $\mathrm{R}$ and the solvent $\mathrm{S}_{2}$ and the operating cost concerning the solvent $S_{2}$ will be determined for the reaction system of concern according to the choice of $S_{2}$.

The values of decision variables for each subsystem $\tau^{n}, \xi^{n}$ and $\zeta^{n}$ and those of structure variables $\zeta_{0 n}$ $(n=1,2, \ldots, N)$ and $\zeta_{m n}(m=1,2, \ldots, N ; n=1,2$, .., $N+1$ ) which maximize the value of $J$ must be sought to determine the optimal flow pattern of the solvent $\mathrm{S}_{2}$.

Though the problem under consideration concerns process design, the fixed cost is not included in the objective function in Eq. (19). The fixed cost will

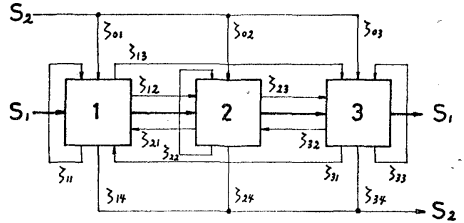

Fig. 2 The structure of three-stage extractive reactors

primarily depend on the total volume of reactors and the total length of the piping network. The optimal value of each reactor volume will primarily be determined by finding a point of compromise between the increase in yield and the decrease in fixed cost without having any significant effect on the solvent flow pattern. On the other hand, the fixed cost of the piping will become neither so large nor so small. Each reactor requires at least one solvent flow and in the optimal solvent flow pattern the most preferable of the possible solvent flows will usually be chosen for each reactor. Therefore, it can be expected that the effect of the fixed cost on the optimal solvent flow pattern will be small relative to that of the other costs. These are the reasons why the fixed cost was not taken into account.

\section{Procedure for Optimization}

Substituting Eqs. (11)-(14) into Eqs. (7)'-(10) yields

$$
f(x, u)=0
$$

where

$$
\begin{aligned}
\boldsymbol{f}= & \left(f_{1}^{1}, f_{2}^{1}, f_{3}^{1}, f_{4}^{1}, \cdots, f_{1}^{N}, f_{2}^{N}, f_{3}^{N}, f_{4}^{N}\right) \\
\boldsymbol{x}= & \left(x_{1}^{1}, x_{2}^{1}, y_{1}^{1}, y_{2}^{1}, \cdots, x_{1}^{N}, x_{2}^{N}, y_{1}^{N}, y_{2}^{N}\right) \\
\boldsymbol{u}= & \left(\tau^{1}, \xi^{1}, \zeta^{1}, \cdots, \tau^{N}, \xi^{N}, \zeta^{N}, \zeta_{01}, \cdots, \zeta_{0 N},\right. \\
& \left.\zeta_{11}, \cdots, \zeta_{1, N+1}, \cdots, \zeta_{N 1}, \cdots, \zeta_{N, N+1}\right)
\end{aligned}
$$

and $(\cdot)^{\mathrm{T}}$ denotes the transpose of a vector. $\boldsymbol{f}$ and $\boldsymbol{x}$ are $4 N$-dimensional vectors while $\boldsymbol{u}$ is a $N(N+5)$ dimensional vector which is the resultant of a $3 N$ dimensional decision vector and an $N(N+2)$-dimensional structure vector.

A set of $2 N$ equalities in (15) and (16) and a set of $N(N+3)$ inequalities in (17) and (18) are linear with respect to $u$ and can be written as

$$
\begin{aligned}
& \boldsymbol{A}_{e} \cdot \boldsymbol{u}-\boldsymbol{b}_{\boldsymbol{e}}=\mathbf{0} \\
& \boldsymbol{A}_{i} \cdot \boldsymbol{u}-\boldsymbol{b}_{i} \leq \mathbf{0}
\end{aligned}
$$

where $\boldsymbol{A}_{e}$ is a $2 N \times N(N+5)$ matrix, $\boldsymbol{A}_{i}$ an $N(N+3)$ $\times N(N+5)$ matrix, $\boldsymbol{b}_{e}$ a $2 N$-vector and $\boldsymbol{b}_{i}$ an $N(N+3)$ vector.

Though the right-hand side of Eq. (19) depends on $\boldsymbol{x}$ and $\boldsymbol{u}, J$ can be regarded as a function of $\boldsymbol{u}$ alone since $\boldsymbol{x}$ can be eliminated by use of Eq. (20). Therefore, we can write

$$
J(\boldsymbol{u})=G(\boldsymbol{x}, \boldsymbol{u})
$$

After all, the optimization problem of concern reduces to a nonlinear programming problem in which 


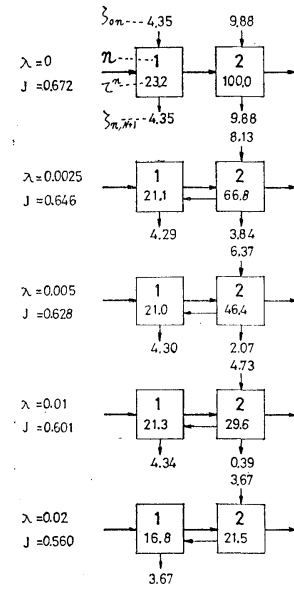

Fig. 3 Optimal solvent flow patterns for two-stage extractive reactors

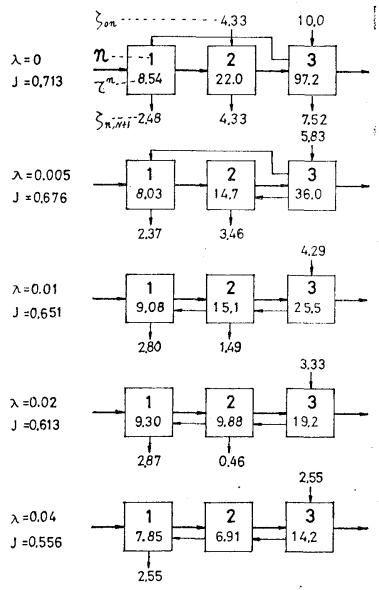

Fig. 4 Optimal solvent flow patterns for three-stage extractive reactors

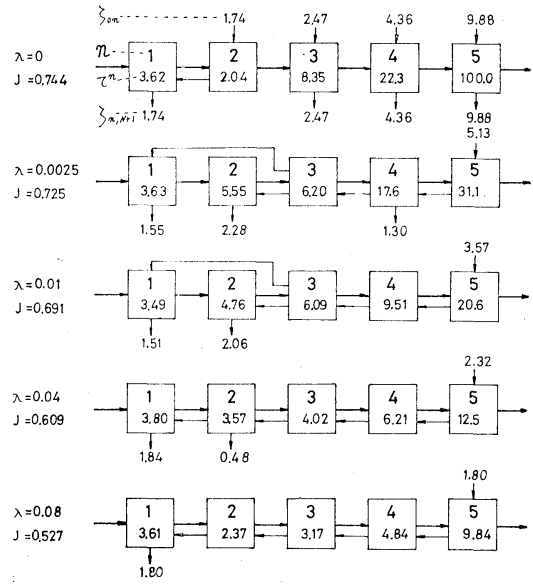

Fig. 5 Optimal solvent flow patterns for five-stage extractive reactors

a nonlinear objective function must be maximized subject to linear constraints. So, if the value of $\mathrm{d} J / \mathrm{d} u$ can readily be evaluated, the method of Goldfarb et al. ${ }^{11}$ can be used for the optimization.

Taking the derivative of both sides of Eq. (23) yields

$$
\frac{\mathrm{d} J}{\mathrm{~d} \boldsymbol{u}}=\frac{\partial G}{\partial \boldsymbol{x}} \cdot \frac{\mathrm{d} \boldsymbol{x}}{\mathrm{d} \boldsymbol{u}}+\frac{\partial G}{\partial \boldsymbol{u}}
$$

where the derivative of a scalar with respect to a column-vector is defined as a row-vector.

By differentiating both sides of Eq. (20) with respect to $\boldsymbol{u}$,

$$
\frac{\partial \boldsymbol{f}}{\partial \boldsymbol{x}} \cdot \frac{\mathrm{d} \boldsymbol{x}}{\mathrm{d} \boldsymbol{u}}+\frac{\partial \boldsymbol{f}}{\partial \boldsymbol{u}}=\mathbf{0}
$$

It is evident from Eqs. (7)'-(10)' that Eq. (20) can be rewritten as

$$
f(x, u)=F(u) x-c=0
$$

Hence, we have

$$
\frac{\partial \boldsymbol{f}}{\partial \boldsymbol{x}}=\boldsymbol{F}(\boldsymbol{u})
$$

Whenever the linear equation (26) about $\boldsymbol{x}$ has a

unique solution, $\boldsymbol{F}(\boldsymbol{u})$ must be nonsingular and consequently there exists its inverse. Hence, we have from Eqs. (25) and (27)

$$
\frac{\mathrm{d} \boldsymbol{x}}{\mathrm{d} \boldsymbol{u}}=-[\boldsymbol{F}(\boldsymbol{u})]^{-1} \cdot \frac{\partial \boldsymbol{f}}{\partial \boldsymbol{u}}
$$

and substituting Eq. (28) into Eq. (24) yields

$$
\frac{\mathrm{d} J}{\mathrm{~d} \boldsymbol{u}}=-\frac{\partial G}{\partial \boldsymbol{x}}[\boldsymbol{F}(\boldsymbol{u})]^{-1} \cdot \frac{\partial \boldsymbol{f}}{\partial \boldsymbol{u}}+\frac{\partial G}{\partial \boldsymbol{u}}
$$

Thus, the computation algorithm for the optimization becomes

Step 1. Choose an initial value of $\boldsymbol{u}$ which satisfies the constraints (21) and (22).

Step 2. Compute the inverse of $\boldsymbol{F}(\boldsymbol{u}),[\boldsymbol{F}(\boldsymbol{u})]^{-1}$.

Step 3. Compute $\boldsymbol{x}=-[\boldsymbol{F}(\boldsymbol{u})]^{-1} \cdot \boldsymbol{c}$

Step 4. Compute $\partial \boldsymbol{f} / \partial \boldsymbol{u}, \partial G / \partial \boldsymbol{u}$ and $\partial G / \partial \boldsymbol{x}$.

Step 5. Compute $\mathrm{d} J / \mathrm{d} \boldsymbol{u}$ from Eq. (29).

Step 6. Stop the procedure if the terminating condition is satisfied, otherwise modify the value of $\boldsymbol{u}$ subject to the linear constraints (21) and (22) according to the method of Goldfarb et al. and return to Step 2.

\section{Optimal Solvent Flow Pattern}

The optimal solvent flow pattern was determined according to the procedure previously described. Figures 3, 4 and 5 show those obtained for $N=2,3$ and 5 , respectively. The optimal value of $\xi^{n}$ always became equal to its upper bound which is specified as 100 in (18). This is a very natural consequence of the fact that the mass transfer rate from the reactive to the extractive phase is desired to be as large as possible.

It is seen in Figs. 3, 4 and 5 that the optimal solvent flow pattern has the following basic features.

(i) Every subsystem has only one inflow of $S_{2}$ and in addition it is either an outflow from a posterior subsystem or a fresh feed flow. The inflow of $S_{2}$ into a subsystem is neither an outflow from an anterior subsystem nor a recycle flow from the exit of the subsystem. Thus, we have

$$
\zeta_{m n}=0 . \quad m \leq n, \quad m \neq 0
$$

(ii) The fresh feed of $S_{2}$ is only fed to the final subsystem unless the value of $\lambda$ is equal to zero.

(iii) The optimal solvent flow pattern is generally very close to the crosscurrent pattern if the value of $\lambda$ is small. But, there may exist some difference between them even if the value of $\lambda$ is equal to zero. That is, the inflow of $S_{2}$ into the first subsystem is usually not fresh feed flow but the outflow from a posterior subsystem except for the case of $N=2$.

(iv) The optimal solvent flow pattern always becomes the countercurrent pattern when the value of $\lambda$ is increased enough.

The relation between the maximal value of $J$ and 
number of subsystems $N$ is shown in Fig. 6 for several values of $\lambda$. As $N$ increases, the maximal value of $J$ increases rapidly for small $N$ but slowly for large $N$. If, however, the fixed cost is taken into account, the maximal value of $J$ will tend to decrease as $N$ increases much and in consequence an optimal value of $N$ will appear.

\section{Discussion}

If the basic features of the optimal solvent flow pattern described in the last section be the general rules, then we can reduce the number of structure variables to be involved. Such a reduction will be effective in seeking the optimal solvent flow pattern, especially when $N$ is very large. Unfortunately, there is no guarantee that these features are the general rules. Some qualitative considerations, however, will to a certain extent assure us of their validity.

The flow of $\mathrm{S}_{2}$ fed to a certain subsystem can be either fresh feed, the outflow from an anterior or a posterior subsystem, or the recycle flow of the subsystem. It will depend on the following four factors which of them is most favorable:

(i) the cost depending on the fresh feed of $S_{2}$,

(ii) the extraction efficiency,

(iii) the recovery of the reactant A contained in the outflow of $\mathrm{S}_{2}$,

(iv) the conversion of the desired product $\mathrm{R}$ into the wasteful product $S$ by the reuse of $S_{2}$ containing $R$.

The fresh feed is most favorable with respect to the second factor while it is quite unfavorable with respect to the first factor. Therefore, if $\lambda=0$, the fresh feed generally becomes the flow of $S_{2}$ into each subsystem, but an outflow from a posterior subsystem often replaces it because of the third factor.

If $\lambda \neq 0$, the reuse of an outflow from any subsystem is geneally preferable to the use of fresh feed, and the outflow from a posterior subsystem is adopted because it is more advantageous than that from an anterior subsystem or recycle flow with respect to the second factor. The final subsystem has, however, no posterior subsystem, and in addition at least one subsystem must be fed the fresh feed. Thus, the fresh feed is only fed to the final subsystem.

As $\lambda$ increases, it becomes important to decrease the amount of $S_{2}$, used because of the first factor. This is probably the reason why the countercurrent pattern becomes optimal when $\lambda$ is large, since the solvent $S_{2}$ is most efficiently reused in this pattern.

In contrast to this, as $\lambda$ decreases, it becomes more advantageous to increase the amount of the desired product $R$ contained in the exhaust of $S_{2}$ than to decrease the amount of $S_{2}$ used. Therefore, in such a situation, the second, the third and the fourth factor become important. Of these, the second factor is

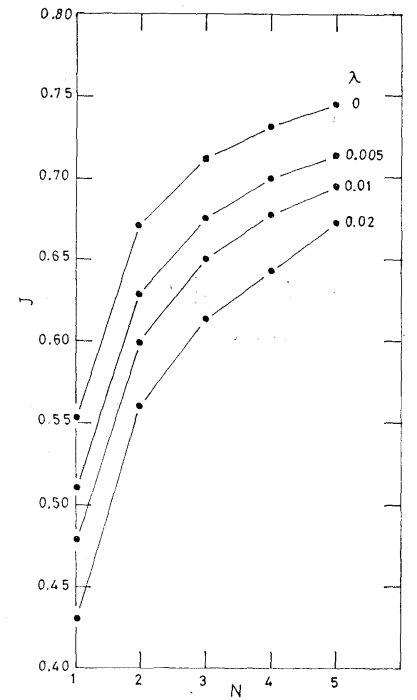

Fig. 6 Effect of $N$ on maximal value of $J$

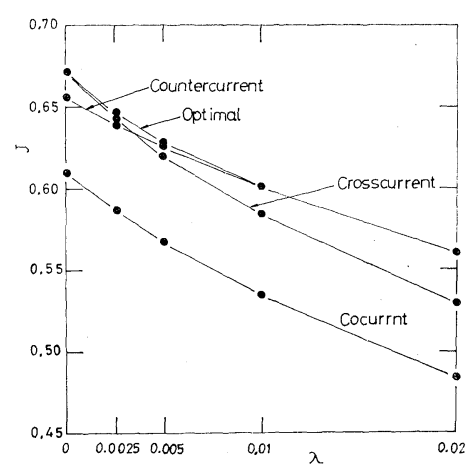

Fig. 7 Effect of $\lambda$ on maximal value of $J$ for $N=2$

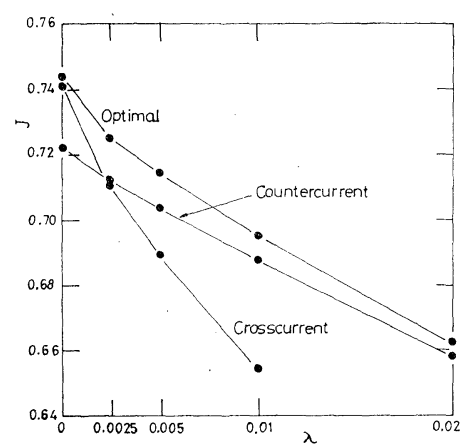

Fig. 8 Effect of $\lambda$ on maximal value of $J$ for $N=5$

usually most dominant. Though the fresh feed is most favorable with respect to the second factor, the outflow from a posterior subsystem assigned the larger subsystem number is more favorable than that assigned the smaller number with respect to the second and the fourth factor. Therefore, as $\lambda$ decreases, the optimal pattern shifts from the countercurrent pattern and approaches the crosscurrent pattern. There may exist, however, some differences between the optimal pattern and the crosscurrent pattern even when $\lambda=0$. This is probably, as already pointed out, due to the fact that the third factor becomes more dominant than the second factor in such a situation. 
The performance of the optimal pattern relative to those of three typical solvent flow patterns, i.e. the crosscurrent, the countercurrent and the cocurrent patterns, is shown in Fig. 7 for $N=2$ and in Fig. 8 for $N=5$. As $\lambda$ increases, the crosscurrent pattern becomes disadvantageous while the countercurrent pattern becomes advantageous. The performance of the cocurrent pattern is always very poor. This pattern is obviously disadvantageous with respect to the second and the fourth factor because the solvent $\mathrm{S}_{2}$ always flows from the anterior subsystem to the posterior subsystem.

The performance of the reactor without extraction is compared with that of the extractive reactor to assure the advantage of the latter. The optimal type of reactor without extraction is the plug flow reactor for the reaction under consideration.

The yield of the desired product $\mathrm{R}$ is

$$
x_{\mathrm{R}}=\frac{k}{2 \sqrt{\alpha^{2}-\beta}}\left(e^{r t}-e^{r^{\prime} t}\right)
$$

where $t$ is the residence time and

$$
\begin{aligned}
2 \alpha & =k+k^{\prime}+k^{\prime \prime}=k\left(1+\kappa_{\mathrm{R}}+\kappa_{\mathrm{C}}\right) \\
\beta & =k k^{\prime \prime}=k^{2} \kappa_{\mathrm{C}} \\
r & =-\alpha+\sqrt{\alpha^{2}-\beta} \\
r^{\prime} & =-\alpha-\sqrt{\alpha^{2}-\beta}
\end{aligned}
$$

The value of $t$ which maximizes the value of $x_{\mathrm{R}}$ can easily be obtained and becomes

$$
t_{\mathrm{opt}}=\ln \left(r / r^{\prime}\right) /\left(r-r^{\prime}\right)
$$

Substitution of Eq. (32) into Eq. (31) yields the maximal value of $x_{\mathrm{R}}$ which becomes equal to 0.088 for $\kappa_{\mathrm{R}}=9$ and $\kappa_{\mathrm{C}}=1$. This is very small relative to the value of $J$ given in Fig. 6 . Therefore, the effect of the extraction is surely large as long as the suitable set of solvents $S_{1}$ and $S_{2}$ can be found.

\section{Conclusion}

Some basic features of the optimal solvent flow pattern for multistage reactors with extraction were clarified through numerical calculations for parameter values tentatively chosen. Qualitative considerations were also given for elucidating the reason why these features were observed. They suggest that these features will probably be the general properties of the optimal solvent flow pattern.

\section{Acknowledgment}

The authors are grateful to $\mathrm{H}$. UOZU who wrote the subprogram for the method of Goldfarb et al.

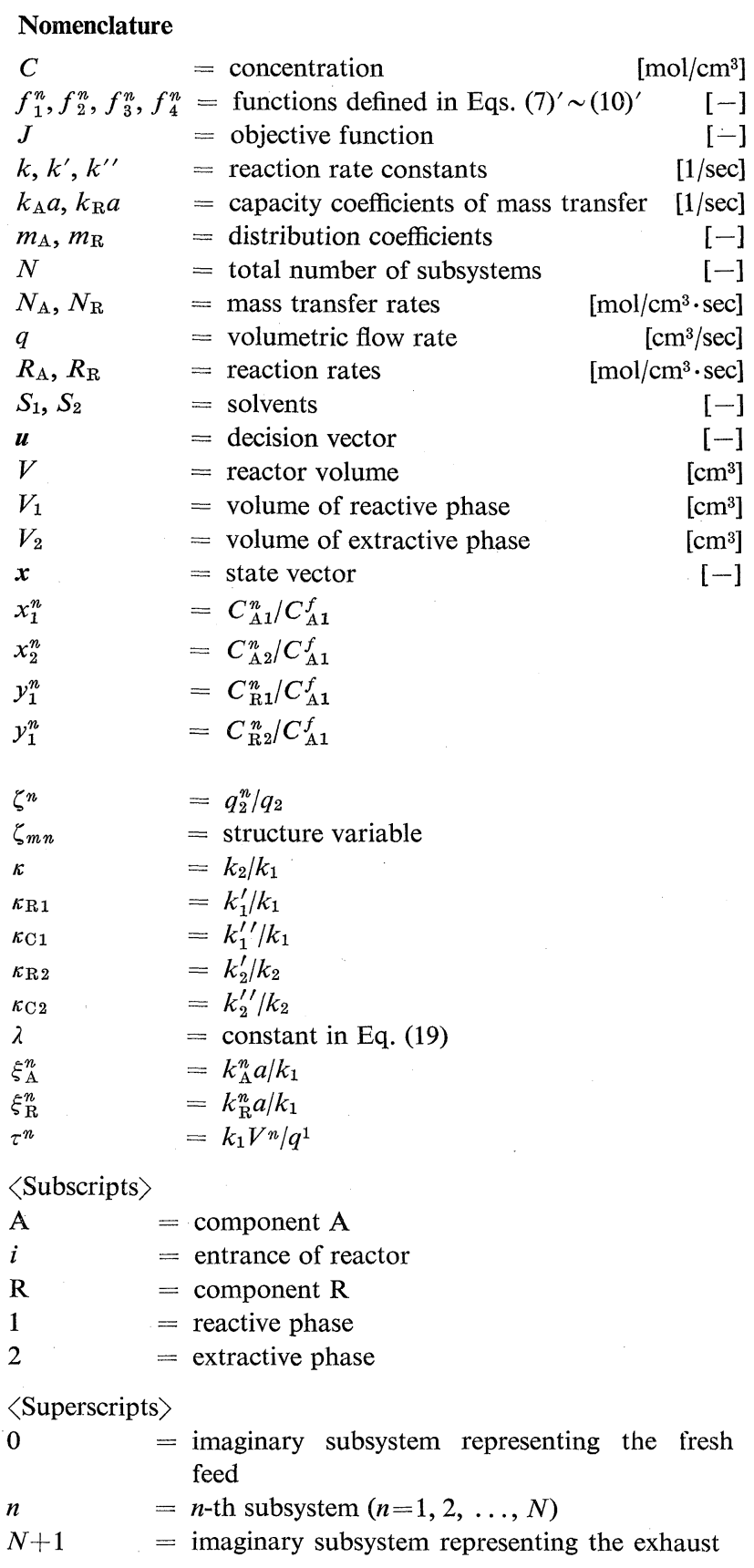

\section{Literature Cited}

1) Goldfarb, D. and L. Lapidus: Ind. Eng. Chem., Fundamentals, 7, 142 (1968)

2) Goto, S. and M. Matsubara: J. Chem. Eng. Japan, 5, 87 (1972)

3) Goto, S. and M. Matsubara: J. Chem. Eng. Japan, 5, 90 (1972)

4) Hendry, J. E., D. F. Rudd and J. D. Seader: AIChE J., 19, 1 (1973)

5) Hofmann, H.: Chem. Eng. Sci., 8, 113 (1958)

6) Ichikawa, A.: Kagaku Kögaku, 36, 1053 (1972)

7) Piret, E. L., W. H. Penny and P. J. Trambouze: AIChE J., 6, 574 (1960)

8) Schoenemann, K.: Chem. Eng. Sci., 14, 39 (1961) 